Journal of Mathematics and Informatics

Vol. 10, 2017, 103-111

ISSN: 2349-0632 (P), 2349-0640 (online)

Published 11 December 2017

www.researchmathsci.org

DOI: http://dx.doi.org/10.22457/jmi.v10a14

Journal of

Mathematics and

Informatics

\title{
Continued Fractions of Ratios of Consecutive Polygonal Numbers
}

\author{
A.Gnanam $^{1}$ and S.Krithika ${ }^{2}$ \\ ${ }^{1}$ Department of Mathematics, Government Arts College \\ Trichy-22, Tamilnadu, India. Email: gnaanam@yahoo.com \\ ${ }^{2}$ Department of Mathematics, Seethalakshmi Ramaswami College \\ Trichy-2, Tamilnadu, India. Email:krithikasrc@gmail.com
}

Received 2 November 2017; accepted 5 December 2017

Abstract. In this paper the patterns of continued fractions of ratios of polygonal numbers for consecutive sizes have been identified.

Keywords: Continued fractions, Simple continued fraction, Euclidean algorithm, Polygonal numbers.

AMS Mathematics Subject Classification(2010): 11A05, 11A55, 11Y65, 40A15, 30B70

\section{Notations}

1. $\left[a_{0}, a_{1}, a_{2}, a_{3}, \cdots a_{n}\right]$ :Continued fraction expansion.

2. $\left[x_{k}\right] \quad$ : Integer part of $x_{k}$

3. $P_{d}(n) \quad$ : Polygonal number of order $\mathrm{d}$ and rank $\mathrm{n}$.

\section{Introduction}

The ancient Greek mathematician Diophantos was one of the first to study polygonal numbers. A polygonal number can be defined as a sum of equidistant dots used to represent a polygon of a certain size. For example, if you have a square number with rank one it is one, rank two is four because you expand the length and width by one dot each and fill in the outer layer, then rank three would be nine and it continues in this fashion. The rank of a polygonal numbers is the number of dots on a side of the outermost layer of the polygonal number. This holds true for all polygonal numbers.

A polygonal number is denoted by $P_{d}(n)$ where $\mathrm{d}$ is the number of sides to the corresponding polygon and $\mathrm{n}$ is the rank, or order, of the polygonal number. For instance $P_{5}(4)$ would be a pentagonal number with rank four. All polygonal numbers with rank one equals one, and all polygonal numbers of rank two are equal to the number of sides on the corresponding polygon.
$P_{3}(3)=6$
$P_{4}(3)=9 P_{5}(3)=12$ 
A.Gnanam and S.Krithika
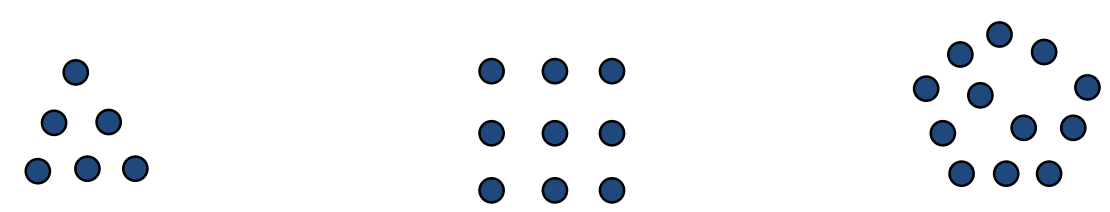

Figure 1:

Furthermore, you can find any polygonal number by using the formula $P_{d}(n)=\frac{(d-2) n^{2}+(4-n) n}{2}$. Note that $d, n \in N$ and, since less than three sides would not form a polygon.

Table 1: Values of some polygonal numbers

\begin{tabular}{|c|c|c|c|c|c|c|c|c|c|c|c|c|}
\hline $\begin{array}{c}\text { Sides } \\
\text { d }\end{array}$ & \multicolumn{10}{|c|}{ Rank of polygonal numbers } \\
\hline $\mathbf{3}$ & 1 & $\mathbf{2}$ & $\mathbf{3}$ & $\mathbf{4}$ & $\mathbf{5}$ & $\mathbf{6}$ & $\mathbf{7}$ & $\mathbf{8}$ & $\mathbf{9}$ & $\mathbf{1 0}$ & $\mathbf{1 1}$ & $\mathbf{1 2}$ \\
\hline $\mathbf{4}$ & 1 & 4 & 9 & 16 & 25 & 36 & 49 & 64 & 81 & 100 & 121 & 144 \\
\hline $\mathbf{5}$ & 1 & 5 & 12 & 22 & 35 & 51 & 70 & 92 & 117 & 145 & 176 & 210 \\
\hline $\mathbf{6}$ & 1 & 6 & 15 & 28 & 45 & 66 & 91 & 120 & 153 & 190 & 231 & 276 \\
\hline $\mathbf{7}$ & 1 & 7 & 18 & 34 & 55 & 81 & 112 & 148 & 189 & 235 & 286 & 342 \\
\hline $\mathbf{8}$ & 1 & 8 & 21 & 40 & 65 & 96 & 133 & 176 & 225 & 280 & 341 & 408 \\
\hline $\mathbf{9}$ & 1 & 9 & 24 & 46 & 75 & 111 & 154 & 204 & 261 & 325 & 396 & 474 \\
\hline $\mathbf{1 0}$ & 1 & 10 & 27 & 52 & 85 & 126 & 175 & 232 & 297 & 370 & 451 & 540 \\
\hline $\mathbf{1 1}$ & 1 & 11 & 30 & 58 & 95 & 141 & 196 & 260 & 333 & 415 & 506 & 606 \\
\hline $\mathbf{1 2}$ & 1 & 12 & 33 & 64 & 105 & 156 & 217 & 288 & 369 & 460 & 561 & 672 \\
\hline $\mathbf{1 3}$ & 1 & 13 & 36 & 70 & 115 & 171 & 238 & 316 & 405 & 505 & 616 & 738 \\
\hline $\mathbf{1 4}$ & 1 & 14 & 39 & 76 & 125 & 186 & 259 & 344 & 441 & 550 & 671 & 804 \\
\hline $\mathbf{1 5}$ & 1 & 15 & 42 & 82 & 135 & 201 & 280 & 372 & 477 & 595 & 726 & 870 \\
\hline $\mathbf{1 6}$ & 1 & 16 & 45 & 88 & 145 & 216 & 301 & 400 & 513 & 640 & 781 & 936 \\
\hline $\mathbf{1 7}$ & 1 & 17 & 48 & 94 & 155 & 231 & 322 & 428 & 549 & 685 & 836 & 1002 \\
\hline $\mathbf{1 8}$ & 1 & 18 & 51 & 100 & 165 & 246 & 343 & 456 & 585 & 730 & 891 & 1068 \\
\hline $\mathbf{1 9}$ & 1 & 19 & 54 & 106 & 175 & 261 & 364 & 484 & 621 & 775 & 946 & 1134 \\
\hline $\mathbf{2 0}$ & 1 & 20 & 57 & 112 & 185 & 276 & 385 & 512 & 657 & 820 & 1001 & 1200 \\
\hline $\mathbf{2 1}$ & 1 & 21 & 60 & 118 & 195 & 291 & 406 & 540 & 693 & 865 & 1056 & 1266 \\
\hline $\mathbf{2 2}$ & 1 & 22 & 63 & 124 & 205 & 306 & 427 & 568 & 729 & 910 & 1111 & 1332 \\
\hline $\mathbf{2 3}$ & 1 & 23 & 66 & 130 & 215 & 321 & 448 & 596 & 765 & 955 & 1166 & 1398 \\
\hline $\mathbf{2 4}$ & 1 & 24 & 69 & 136 & 225 & 336 & 469 & 624 & 801 & 1000 & 1221 & 1464 \\
\hline $\mathbf{2 5}$ & 1 & 25 & 72 & 142 & 235 & 351 & 490 & 652 & 837 & 1045 & 1276 & 1530 \\
\hline $\mathbf{2 6}$ & 1 & 26 & 75 & 148 & 245 & 366 & 511 & 680 & 873 & 1090 & 1331 & 1596 \\
\hline $\mathbf{2 7}$ & 1 & 27 & 78 & 154 & 255 & 381 & 532 & 708 & 909 & 1135 & 1386 & 1662 \\
\hline $\mathbf{2 8}$ & 1 & 28 & 81 & 160 & 265 & 396 & 553 & 736 & 945 & 1180 & 1441 & 1728 \\
\hline $\mathbf{2 9}$ & 1 & 29 & 84 & 166 & 275 & 411 & 574 & 764 & 981 & 1225 & 1496 & 1794 \\
\hline $\mathbf{3 0}$ & 1 & 30 & 87 & 172 & 285 & 426 & 595 & 792 & 1017 & 1270 & 1551 & 1860 \\
\hline
\end{tabular}


Continued Fractions of Ratios of Consecutive Polygonal Numbers

Continued fraction plays an important role in number theory. It is used to represent the rational numbers to an another form by using Euclidean algorithm.

An expression of the form

$$
\frac{p}{q}=a_{0}+\frac{b_{0}}{a_{1}+\frac{b_{1}}{a_{2}+\frac{b_{2}}{a_{3}+\frac{b_{3}}{\ddots}}}}
$$

where $a_{i}, b_{i}$ are real or complex numbers is called a continued fraction.

An expression of the form

$$
\frac{p}{q}=a_{0}+\frac{1}{a_{1}+\frac{1}{a_{2}+\frac{1}{a_{3}+\frac{1}{\ddots}}}}
$$

where $b_{i}=1 \forall i$, and $a_{0}, a_{1}, a_{2}, \cdots \quad$ are each positive integers is called a simple continued fraction.

The continued fraction is commonly expressed as

$$
\frac{p}{q}=a_{0}+\frac{1}{a_{1}+} \frac{1}{a_{2}+} \frac{1}{a_{3}+} \cdots \text { or simply as }\left[a_{0}, a_{1}, a_{2}, a_{3}, \cdots\right]
$$

The elements $a_{0}, a_{1}, a_{2}, a_{3}, \cdots$ are called the partial quotients. If there are finite number of partial quotients, we call it finite simple continued fraction, otherwise it is infinite.

The continued fraction expansion of $22 / 7$ is $[3 ; 7]$. Here $a_{0}=3$.

\section{The continued fraction algorithm}

Suppose we wish to find continued fraction expansion of $x \in R$.

Let $x_{0}=x$ and set $a_{0}=\left[x_{0}\right]$.

Define $x_{1}=\frac{1}{x_{0}-\left[x_{0}\right]}$ and set $a_{1}=\left[x_{1}\right]$ 
A.Gnanam and S.Krithika

and $x_{2}=\frac{1}{x_{1}-\left[x_{1}\right]} \Rightarrow a_{2}=\left[x_{2}\right], \ldots ., x_{k}=\frac{1}{x_{k-1}-\left[x_{k-1}\right]} \Rightarrow a_{k}=\left[x_{k}\right], \ldots$.

This process is continued infinitely or to some finite stage till an $x_{i} \in N$ exists such that $a_{i}=\left[x_{i}\right]$.

\section{Examples}

1. Continued fraction expansion of $414 / 283=1.4629$ is $[1 ; 2,6,4,5]$

2. Continued fraction expansion of $\sqrt{3}$ and $\sqrt{7}$ are $[1 ; 1,2,1,2,1,2, \ldots]$ and $[2 ; 1,1,1,4,1,1,1,4, \ldots]$. Which are known as periodic continued fractions. The above periodic continued fractions are also denoted by $[1 ; \overline{1,2}]$ and $[2 ; \overline{1,1,1,4]}$.

In this paper we try to find some patterns of ratios of consecutive polygonal numbers of different sides and different orders using continued fractions.

Theorem 1. The continued fraction of ratio of consecutive polygonal number of rank 2 is $[0 ; 1, d]$ where $d$ is the sides of a polygon and $d \geq 3$.

In other words $\frac{P_{d}(2)}{P_{d+1}(2)}=[0 ; 1, d]$, where $d \geq 3$.

Proof: Take $d=3$.

Therefore $\frac{P_{d}(2)}{P_{d+1}(2)}=\frac{3}{4}$.

Using continued fraction algorithm,

Take $x_{0}=\frac{3}{4}$, so $a_{0}=0$. Then $x_{1}=\frac{1}{x_{0}-\left[x_{0}\right]}=\frac{3}{4}=1+\frac{1}{3} \Rightarrow a_{1}=1$.

$x_{2}=\frac{1}{x_{1}-\left[x_{1}\right]}=3 \Rightarrow a_{2}=3$.

Therefore $\frac{P_{3}(2)}{P_{4}(2)}=\frac{3}{4}=[0 ; 1,3]$.

The result is true when $d=3$.

Assume the result is true for $d=k-1, k \geq 4$.

Therefore $\frac{P_{k-1}(2)}{P_{k}(2)}=[0 ; 1, k-1]$.

We prove the result for $d=k$.

$\frac{P_{k}(2)}{P_{k+1}(2)}=\frac{(k-2) 4+(k-2) 2}{(k-1) 4+(3-k) 2}=\frac{(k-2) 2+(k-2)}{(k-1) 2+(3-k)}=\frac{k}{k+1}$. 


\section{Continued Fractions of Ratios of Consecutive Polygonal Numbers}

Take

$$
x_{0}=\frac{k}{k+1}
$$

so $a_{0}=0$.

Then $x_{1}=\frac{1}{x_{0}-\left[x_{0}\right]}=\frac{k+1}{k}=1+\frac{1}{k} \Rightarrow a_{1}=1$

$x_{2}=\frac{1}{x_{1}-\left[x_{1}\right]}=k \Rightarrow a_{2}=k$.

Therefore $\frac{P_{k}(2)}{P_{k+1}(2)}=[0 ; 1, k]$.

Hence by induction the result is true for all values of $\mathrm{d}$, where $d \geq 3$.

Theorem 2. The continued fraction of ratio of consecutive polygonal number of rank 3 is $[0 ; 1, d-1]$ where $d$ is the sides of a polygon and $d \geq 3$.

In other words $\frac{P_{d}(3)}{P_{d+1}(3)}=[0 ; 1, d-1]$, where $d \geq 3$.

Proof: Similar to the proof of theorem 1.

Theorem 3. The continued fraction of ratio of consecutive polygonal number of side $\geq 4$ and order $\geq 3$ is

$\left[0 ; 1, d-2, \frac{R-1}{2}\right]$ if $R$ is odd and $\left[0 ; 1, d-2, \frac{R-2}{2}, 2\right]$ if $R$ is even.

In other words $\frac{P_{d}(R)}{P_{d+1}(R)}=\left\{\begin{array}{l}{\left[0 ; 1, d-2, \frac{R-1}{2}\right] \text { if } R \text { is odd }} \\ {\left[0 ; 1, d-2, \frac{R-2}{2}, 2\right] \text { if } R \text { is even }}\end{array}\right.$

\section{Proof:}

Case (i): When $R$ is odd ie., $R=2 n+1, n \geq 2$.

Take $d=3$ and $R=5$.

Therefore $\frac{P_{3}(5)}{P_{4}(5)}=\frac{15}{25}$.

Using continued fraction algorithm,

Take $x_{0}=\frac{15}{25}$, so $a_{0}=0$. Then $x_{1}=\frac{1}{x_{0}-\left[x_{0}\right]}=\frac{25}{15}=1+\frac{10}{15} \Rightarrow a_{1}=1$.

$x_{2}=\frac{1}{x_{1}-\left[x_{1}\right]}=\frac{15}{10}=1+\frac{5}{10} \Rightarrow a_{2}=1$. 


\section{A.Gnanam and S.Krithika}

$x_{3}=\frac{1}{x_{2}-\left[x_{2}\right]}=\frac{10}{5}=2 \Rightarrow a_{3}=2$

Therefore $\frac{P_{3}(5)}{P_{4}(5)}=\frac{15}{25}=[0 ; 1,1,2]$.

Therefore the result is true for $d=3$ and $R=5$.

Assume the result is true for $d=k-1$ and $R=2 n-1$.

$$
\frac{P_{k-1}(2 n-1)}{P_{k}(2 n-1)}=\left[0 ; 1, k-3, \frac{2 n-2}{2}\right]=[0 ; 1, k-3, n-1] \text {. }
$$

Prove the result is true for $d=k$ and $R=2 n+1$.

Therefore

$$
\begin{aligned}
& \quad \frac{P_{k}(2 n+1)}{P_{k+1}(2 n+1)}=\frac{(k-2)(2 n+1)^{2}+(4-k)(2 n+1)}{(k-1)(2 n+1)^{2}+(3-k)(2 n+1)}=\frac{(k-2)(2 n+1)+(4-k)}{(k-1)(2 n+1)+(3-k)} \\
& =\frac{2 k n-4 n+2}{2 k n-2 n+2} \\
& =\frac{k n-2 n+1}{k n-n+1} \\
& \text { Therefore } \frac{P_{k}(2 n+1)}{P_{k+1}(2 n+1)}=\frac{k n-2 n+1}{k n-n+1}
\end{aligned}
$$

Using continued fraction algorithm,

$$
\begin{aligned}
& \quad \text { Take } x_{0}=\frac{k n-2 n+1}{k n-n+1} \Rightarrow \\
& x_{1}=\frac{1}{x_{0}-\left[x_{0}\right]}=\frac{k n-n+1}{k n-2 n+1}=1+\frac{n}{k n-2 n+1} \Rightarrow a_{1}=1 . \\
& x_{2}=\frac{1}{x_{1}-\left[x_{1}\right]}=\frac{k n-2 n+1}{n}=k-2+\frac{1}{n} \Rightarrow a_{2}=k-2 . \\
& x_{3}=\frac{1}{x_{2}-\left[x_{2}\right]}=n \Rightarrow a_{3}=n
\end{aligned}
$$

Therefore $\frac{P_{k}(2 n+1)}{P_{k+1}(2 n+1)}=[0 ; 1, k-2, n]$.

Hence by induction the result is true for all value of $d$ and $R$ when $R$ is odd and $d \geq 3$.

Case (ii): When $R$ is even ie., $R=2 n, n \geq 2$.

Take $d=3$ and $R=4$.

Therefore $\frac{P_{3}(4)}{P_{4}(4)}=\frac{10}{16}$.

Using continued fraction algorithm, 
Continued Fractions of Ratios of Consecutive Polygonal Numbers

Take $x_{0}=\frac{10}{16}$, so $a_{0}=0$. Then $x_{1}=\frac{1}{x_{0}-\left[x_{0}\right]}=\frac{16}{10}=1+\frac{6}{10} \Rightarrow a_{1}=1$.

$x_{2}=\frac{1}{x_{1}-\left[x_{1}\right]}=\frac{10}{6}=1+\frac{4}{6} \Rightarrow a_{2}=1$.

$x_{3}=\frac{1}{x_{2}-\left[x_{2}\right]}=\frac{6}{4}=1+\frac{2}{4} \Rightarrow a_{3}=1$

$x_{4}=\frac{1}{x_{3}-\left[x_{3}\right]}=\frac{4}{2}=2 \Rightarrow a_{4}=2$

Therefore $\frac{P_{3}(4)}{P_{4}(4)}=\frac{10}{16}=[0 ; 1,1,1,2]$.

Therefore the result is true for $d=3$ and $R=4$.

Assume the result is true for $d=k-1$ and $R=2 n-1$.

$$
\frac{P_{k-1}(2 n-1)}{P_{k}(2 n-1)}=\left[0 ; 1, k-3, \frac{2 n-2}{2}\right]=[0 ; 1, k-3, n-1] \text {. }
$$

Prove the result is true for $d=k$ and $R=2 n$.

Therefore $\frac{P_{k}(2 n)}{P_{k+1}(2 n)}=\frac{(k-2)(2 n)^{2}+(4-k)(2 n)}{(k-1)(2 n)^{2}+(3-k)(2 n)}=\frac{(k-2)(2 n)+(4-k)}{(k-1)(2 n)+(3-k)}$

$=\frac{2 k n-4 n-k+4}{2 k n-2 n-k+3}$

Therefore $\quad \frac{P_{k}(2 n)}{P_{k+1}(2 n)}=\frac{2 k n-4 n-k+4}{2 k n-2 n-k+3}$

Using continued fraction algorithm,

Take $x_{0}=\frac{2 k n-4 n-k+4}{2 k n-2 n-k+3} \Rightarrow a_{0}=0$. Then

$$
\begin{aligned}
& x_{1}=\frac{1}{x_{0}-\left[x_{0}\right]}=\frac{2 k n-2 n-k+3}{2 k n-4 n-k+4}=1+\frac{2 n-1}{2 k n-4 n-k+4} \Rightarrow a_{1}=1 . \\
& x_{2}=\frac{1}{x_{1}-\left[x_{1}\right]}=\frac{2 k n-4 n-k+4}{2 n-1}=k-2+\frac{2}{2 n-1} \Rightarrow a_{2}=k-2 . \\
& x_{3}=\frac{1}{x_{2}-\left[x_{2}\right]}=\frac{2 n-1}{2} \Rightarrow n-1+\frac{1}{2} a_{3}=n-1 \\
& x_{4}=\frac{1}{x_{3}-\left[x_{3}\right]}=2 \Rightarrow a_{4}=2 \\
& \text { Therefore } \frac{P_{k}(2 n)}{P_{k+1}(2 n)}=[0 ; 1, k-2, n-1,2] .
\end{aligned}
$$




\section{A.Gnanam and S.Krithika}

Hence by induction the result is true for all values of $d$ and $R$ when $R$ is even and $d \geq 3$.

Hence $\frac{P_{d}(R)}{P_{d+1}(R)}=\left\{\begin{aligned} & {\left[0 ; 1, d-2, \frac{R-1}{2}\right] \text { if } R \text { is odd } } \\ & {\left[0 ; 1, d-2, \frac{R-2}{2}, 2\right] \text { if } \text { R is even } }\end{aligned}\right.$

\section{Illustration}

The following table gives the patterns of continued fractions of consecutive polygonal numbers of different orders and ranks.

\begin{tabular}{|c|c|}
\hline $\begin{array}{l}\text { Consecutive fractions of polygonal } \\
\text { numbers }\end{array}$ & Continued fraction expansion \\
\hline$\frac{P_{5}(2)}{P_{6}(2)}$ & {$[0 ; 1,5]$} \\
\hline$\frac{P_{12}(2)}{P_{13}(2)}$ & {$[0 ; 1,12]$} \\
\hline$\frac{P_{4}(3)}{P_{5}(3)}$ & {$[0 ; 1,3]$} \\
\hline$\frac{P_{15}(3)}{P_{16}(3)}$ & {$[0 ; 1,14]$} \\
\hline$\frac{P_{9}(5)}{P_{10}(5)}$ & {$[0 ; 1,7,2]$} \\
\hline$\frac{P_{17}(6)}{P_{18}(6)}$ & {$[0 ; 1,15,2,2]$} \\
\hline$\frac{P_{10}(9)}{P_{11}(9)}$ & {$[0 ; 1,8,4]$} \\
\hline$\frac{P_{26}(10)}{P_{271}(10)}$ & {$[0 ; 1,24,4,2]$} \\
\hline$\frac{P_{29}(12)}{P_{30}(12)}$ & {$[0 ; 1,27,5,2]$} \\
\hline
\end{tabular}

\section{Conclusion}

In this paper, we have identified various patterns of continued fractions of ratios of polygonal numbers of consecutive sizes. This work may be extended to higher order figurate numbers like pyramidal numbers.

\section{REFERENCES}

1. George E. Andrews, Number Theory, W.B. Saunders Company. 


\section{Continued Fractions of Ratios of Consecutive Polygonal Numbers}

2. Ivan Niven, Herbert S. Zuckerman, Hugh L. Montgomery, An introduction to theory of numbers, Fifth edition, Wiley Student Edition.

3. Jonathan Browein, Alfvander Poorten, Jeffrey Shallit, Wadim Zudilin, Never ending Fractions An Introduction to continued fractions, Cambridge University Press.

4. http://www.maths.surrey.ac.uk/hostedsites/R.Knott/Figurate/figurate.html\#section3.2.1

5. https://en.wikipedia.org/wiki/Polygonal_number

6. http://oeis.org/wiki/Polygonal_numbers 